\title{
Junge Menschen sprechen über die Einbürgerung: gesellschaftliche Positionierung und formale Staatsbürgerschaft im Übergang ins Erwachsenenalter
}

\author{
Eva Mey*
}

* ZHAW Zürcher Hochschule für Angewandte Wissenschaften, Departement Soziale Arbeit, CH-8037 Zürich,eva.mey@zhaw.ch.

\begin{abstract}
s
Junge Menschen sprechen über die Einbürgerung: gesellschaftliche Positionierung und formale Staatsbürgerschaft im Übergang ins Erwachsenenalter

DerText basiert aufden vorläufigen Befunden aus einer qualitativen Langzeitstudie zur gesellschaftlichen Positionierung von Jugendlichen ausländischer Herkunft in der Schweiz. Er präsentiert drei wesentliche Grundmuster in den Haltungen der Jugendlichen gegenüber einer Einbürgerung in die Schweiz: Anspruch, Irrelevanz und stolzer Verzicht. Die unterschiedlichen Einbürgerungsstrategien werden in ihrer engen Verknüpfung mit der Selbst-Positionierung der jungen Menschen im sozialen Raum sowie als Strategie im Kontext restriktiver und ethnisierter Einbürgerungspolitik diskutiert. Die Deutungen und Strategien der Jugendlichen weisen zum einen auf Mechanismen der Reproduktion wenig privilegierter Positionen im (transnationalen) sozialen Raum über Staatsbürgerschaft hin, zeugen zum anderen aber auch vom Bedeutungswandel und -verlust der Institution Staatsbürgerschaft auf gesellschaftlicher Ebene.

Schlüsselwörter: Zweite Generation, Staatsbürgerschaft, Adoleszenz, soziale Ungleichheit, Langzeitstudie
\end{abstract}

Les jeunes parlent de la naturalisation : le positionnement social et la citoyenneté lors de la transition vers l'âge adulte

Le texte se fonde sur les résultats intermédiaires d'une étude longitudinale qualitative portant sur le positionnement social de jeunes d'origine étrangère en Suisse. Il présente leurs manières de se situer face à la naturalisation en identifiant trois modèles principaux: revendication d'un droit, déni de pertinence et refus orgueilleux. Ces diverses stratégies sont analysées en lien étroit avec leur positionnement dans l'espace social et en tant que stratégie dans le contexte d'une politique de naturalisation restrictive et ethnicisante. Les significations attachées à la naturalisation et les stratégies des jeunes montrent d'une part les mécanismes de reproduction des positions sociales défavorisées dans l'espace social transnational par le biais de la nationalité mais elles témoignent aussi d'autre part de l'érosion voire de la perte de sens de l'institution de la nationalité à l'échelle sociétale.

Mots-clés: Deuxième génération, citoyenneté, adolescence, inégalité sociale, étude longitudinale

\section{Young People Talk about Naturalization: Social Positioning and Citizenship in the} Transition to Adulthood

This paper is based on the intermediate results of a qualitative longitudinal study which aims at analysing how young people of foreign origin in Switzerland position themselves in the host country's society. It presents three basic models with regard to the migrant youths' attitudes towards naturalisation in Switzerland: entitlement claim, irrelevance and proud renunciation. The three models are discussed in terms of their close relationship with the young migrants' social self-positioning as well as in terms of individual strategies against the restrictive and ethnicising Swiss naturalisation policy. The young migrants' interpretations and strategies show for one the mechanisms according to which low social positions in the transnational social space are being reproduced. They also bear witness to the erosion, loss of meaning even, of the institution of citizenship at a societal scale.

Keywords: Second generation, citizenship, adolescence, social inequality, longitudinal study 


\title{
Junge Menschen sprechen über die Einbürgerung: gesellschaftliche Positionierung und formale Staatsbürgerschaft im Übergang ins Erwachsenenalter
}

\author{
Eva Mey*
}

\section{$1 \quad$ Einleitung}

Wer gehört dazu, wer nicht? Politisch-rechtlich wird diese Frage in nationalstaatlich verfassten Gesellschaften über die formale Staatsbürgerschaft ${ }^{1}$ beantwortet: Volle Mitgliedschaft auch im Sinne politisch-rechtlicher Gleichstellung besitzt nur, wer formal Bürgerin bzw. Bürger des betreffenden Staates ist. Staatsbürgerschaft beschreibt im Kern eine rechtliche Beziehung zwischen Individuum und Staat und umfasst bestimmte soziale, politische und bürgerliche Rechte (Marshall 1992; Reichel 2011). In einem erweiterten Verständnis von Staatsbürgerschaft bezieht sich diese nebst den formalen Dimensionen von Status und Rechten auch auf informelle und symbolische Dimensionen wie die ideelle Verbundenheit mit der politischen Gemeinschaft und die konkrete, gelebte Praxis und Partizipation (Mackert 2006; Bloemraad et al. 2008). Um den aktiven, gelebten Aspekt zu verdeutlichen, wird im Englischen auch von active citizenship oder lived citizenship (Lister et al. 2003) gesprochen.

Der Zugang zur formalen Staatsbürgerschaft erfolgt über Geburt oder durch Einbürgerung. Er unterliegt gesetzlichen Regelungen, die Gegenstand nationalstaatlicher Politik sind und liberaler oder restriktiver sein können. Bommes und Halfmann (1994) und andere (für die Schweiz: Wimmer 1998; D’Amato 2008; Wicker 2011) haben herausgearbeitet, wie Zuwanderungs- und Einbürgerungsgesetzgebung historisch bedingt und, als wesentliche Bestandteile der Migrationspolitik eines Staates, eng - und gegenläufig - aufeinander bezogen sind. Eine Liberalisierung der Zuwanderung und mit ihr eine relative Öffnung der «Aussengrenze» der Nation waren immer schon, und sind es auch heute, begleitet vom Bemühen, über eine differenzierte Einbürgerungs- und Integrationsgesetzgebung die für Nationen

ZHAW Zürcher Hochschule für Angewandte Wissenschaften, Departement Soziale Arbeit, CH-8037 Zürich, eva.mey@zhaw.ch.

1 Im weitverzweigten Diskurs zu Staatsbürgerschaft wird häufig auf den englischen Terminus Citizenship zurückgegriffen, der im englischen Sprachgebrauch eine leicht andere Bedeutung hat als im deutschen Sprachgebrauch. Ich verzichte im vorliegenden Text auf den Citizenship-Begriff und verwende stattdessen fast ausschliesslich den Begriff der Staatsbürgerschaft, wobei ich mich in der Regel auf das enge Verständnis (im Sinne von Staatsangehörigkeit) beziehe. Steht der formale Aspekt im Zentrum des jeweiligen Arguments, verwende ich den Begriff formale Staatsbürgerschaft. Zur Begrifflichkeit im Staatsbürgerschafts- bzw. Citizenshipdiskurs vgl. Reichel (2011). 
konstitutive Grenze zwischen «Innen» und «Aussen», die Dynamik von Ein- und Ausschluss, auch ins Innere des nationalen Territoriums zu verlagern: "Jeder Staat errichtet eine begriffliche, legale und ideologische Grenze zwischen Bürgern und Ausländern» (Brubaker in Mackert 2006, 81). Gleichzeitig gerät im Kontext von Globalisierung, abnehmender Autonomie von Nationalstaaten und vermehrter Migration die Institution der Staatsbürgerschaft selber unter Druck und unterliegt einem tiefgreifenden Wandel (Bommes 2004; Mackert und Müller 2007; Bös und Schmid 2012); die eindeutige Anbindung bürgerlicher Rechte an den Nationalstaat wird hinterfragt und gelockert (Soysal 1994).

Die Situation in der Schweiz zeichnet sich durch ein im europäischen Vergleich besonders restriktives Einbürgerungsgesetz aus, insbesondere für die Nachfahren von Zugewanderten, die sogenannte zweite Generation. ${ }^{2}$ Die Einbürgerungsfrage ist stark politisiert und symbolisch aufgeladen (Fibbi et al. 2007; D'Amato 2008), es gilt ein ethnisch geprägtes Verständnis von Zugehörigkeit. Im Grundsatz orientiert am Herkunftsprinzip (ius sanguinis, in Abgrenzung zum liberalen Territorialprinzip des ius soli) wird volle Mitgliedschaft in der politischen Gemeinschaft nur jenen automatisch gewährt, die als Kind von Schweizer Eltern geboren worden sind. Söhne und Töchter aus Zuwandererfamilien, die sogenannte zweite Generation, sind damit in spezifischer Weise vom restriktiven Zugang zur Staatsbürgerschaft betroffen: Hier geboren wie ihre einheimischen Gleichaltrigen, ist ihre politisch-rechtliche Stellung doch eine deutlich andere.

Im Rahmen der klassischen Migrations- und Integrationsforschung ist die Bedeutung von (formaler) Staatsbürgerschaft ein vergleichsweise wenig beleuchtetes Thema. Zwar existiert eine Vielzahl von Studien zu belonging oder Zugehörigkeit als informelle Dimensionen von Staatsbürgerschaft. Die politisch-rechtliche Gleichstellung hingegen als ungleichheitsrelevanter Aspekt im Prozess gesellschaftlicher Positionierung wird selten in den Blick genommen.

Wichtige empirische Anhaltspunkte für den Zusammenhang zwischen (formaler) Staatsbürgerschaft und gesellschaftlicher Stellung liegen jedoch aus der Einbürgerungsforschung vor. Spezifisch für die Schweiz liess sich bisher wiederholt zeigen, dass Eingebürgerte sozial deutlich besser gestellt sind als nicht eingebürgerte Personen (Wanner und Steiner 2012). Das bessere Abschneiden von Eingebürgerten gilt insbesondere in Bezug auf das Bildungsniveau. Hier sind eingebürgerte Jugendliche so erfolgreich, dass sie sogar die gebürtigen Schweizer Jugendlichen übertreffen (u. a. Bolzman et al. 2003; Mey et al. 2005; Fibbi et al. 2007). In Bezug auf die Stellung im Arbeitsmarkt sind die Befunde allerdings weniger ausgeprägt. Anhand

2 Erschwerend kommt hinzu, dass die Einbürgerung in der Schweiz die Einbürgerung auf Gemeindeebene voraussetzt und der Zugang zu den Gemeindebürgerrechten je nach Gemeinde an andere Bedingungen geknüpft ist, was nebst unterschiedlichem sozioprofessionellem Status der Gemeinden und je anderen politischen Kräfteverhältnissen zu den hohen Unterschieden bei Einbürgerungsraten auf kommunaler Ebene beiträgt (Achermann und Gass 2003; Helbling und Kriesi 2004; Witmer 2008). 
von Erwerbslosenquoten zeigen Fibbi et al. (2007) auf, dass das Risiko, keine Arbeit zu finden, für eingebürgerte Jugendliche zwar immer noch geringer ist als für ihre nicht eingebürgerten Gleichaltrigen, doch höher als bei den gebürtigen Schweizer Jugendlichen. Den Jugendlichen ausländischer Herkunft gelingt es weniger gut, ihr Bildungskapital auf dem Arbeitsmarkt zu verwerten - ein Befund, den die Autoren als Hinweis auf die Diskriminierung von Jugendlichen ausländischer Herkunft beim Zugang zum Arbeitsmarkt deuten und darauf zurückführen, dass der Zugang zu Bildung stärker nach universalistischen Prinzipien organisiert ist als jener zu Arbeit (Fibbi et al. 2007). Hier zeigen sich auch Effekte nach Herkunftsnation: Die schlechtesten Chancen, ihre Bildungstitel zu verwerten, haben Jugendliche aus der Türkei und dem ehemaligen Jugoslawien (Mey et al. 2005; Fibbi et al. 2007).

Insgesamt ist mit Blick auf die Forschung zum Zusammenhang zwischen gesellschaftlicher Stellung und Einbürgerung festzuhalten, dass bisher noch kaum gesicherte Befunde zur Richtung der Kausalität vorliegen (Wanner und Steiner 2012). Mit anderen Worten lässt sich aufgrund vorliegender Resultate nicht aussagen, ob eine erfolgreiche Stellung in Bildung und Beruf eine Folge der Einbürgerung oder die Einbürgerung eine Folge der erfolgreichen Stellung in Bildung und Beruf ist. Als Forschungslücke ist auch zu nennen, dass gerade in der Schweiz noch wenig empirisches Wissen zu den subjektiven Beweggründen einer Einbürgerung vorliegt. ${ }^{3}$ Damit gibt es bislang kaum vertiefte Kenntnisse zur Genese individueller Einbürgerungsentscheide, die es erlauben würden, diese als Resultat des Zusammenspiels von politisch-rechtlichen Rahmenbedingungen, sozialer Lage und dem Handeln individueller Akteure beschreiben und verstehen zu können.

Der vorliegende Beitrag stellt vor dem skizzierten Hintergrund die Frage, wie Jugendliche mit Migrationshintergrund über eine Einbürgerung denken, und untersucht, wie ihre Deutungen und Strategien in Bezug auf die formale Staatsbürgerschaft im biografischen Zusammenhang verortet sind. Er tut dies anhand des biografischen Materials, das aus einer laufenden qualitativen Langzeitstudie ${ }^{4}$ bisher vorliegt. Die Studie startete im Jahr 2005 und wird in der Schweizer Agglomerationsgemeinde Emmen ${ }^{5}$ durchgeführt. Im Rahmen dieser Studie sind 34 junge Frauen und Männer

3 Als wichtige Ausnahme zu erwähnen ist hier eine qualitative Studie aus Genf, in welcher der Autor unterschiedliche Logiken in den Motiven für oder gegen eine Einbürgerung bei Jugendlichen der zweiten Generation herausarbeitet und in Bezug zur bisher erreichten sozialen Stellung setzt (Frauenfelder 2007).

4 Finanziert wird die Studie vom Schweizerischen Nationalfonds, in einer früheren Phase war die Eidgenössische Kommission für Migrationsfragen an den Kosten beteiligt. Die Studie wurde zunächst an der Hochschule Luzern und wird heute an der Zürcher Hochschule für Angewandte Wissenschaften unter der Leitung der Autorin durchgeführt.

5 Emmen ist eine Agglomerationsgemeinde im Raum Luzern mit gut 30000 EinwohnerInnen (Zeitpunkt zweite Befragung). Die Gemeinde blickt auf eine blühende Industrievergangenheit zurück, in deren Verlauf sich viele ausländische Arbeitskräfte hier ansiedelten; die Gemeinde spricht von über 100 Ethnien, die heute in Emmen leben. Dem Kontext Emmen kommt für die hier bearbeitete Fragestellung insofern besondere Bedeutung zu, als die Gemeinde während längerer Zeit ein besonders restriktives Einbürgerungsregime und -verfahren aufwies, das nach 
ausländischer Herkunft ${ }^{6}$ im Übergang ins Erwachsenenalter bisher zweimal mittels biografisch-narrativen Interviews ${ }^{7}$ befragt worden, das erste Mal im Alter von rund 16 Jahren, das zweite Mal im Alter von rund 19 Jahren. Die erste Befragung wurde in den Jahren 2005 bis 2007, die zweite in den Jahren 2008 und 2009 durchgeführt. Eine dritte Befragung ist in Vorbereitung. Ziel der Studie ist es, den Wandel und das Zusammenspiel unterschiedlicher Prozesse gesellschaftlicher Positionierung in Bezug auf Bildung/Beruf, soziale Netzwerke und auch Staatsbürgerschaft im Übergang ins Erwachsenenalter sichtbar machen und differenziert analysieren zu können. Der Begriff der Positionierung ist dabei bewusst gewählt, erstens um damit die Besetzung bestimmter Positionen jenseits der Konzepte aus dem (z. T. normativ geprägten) Integrations- und Assimilationsdiskurs zu untersuchen, zweitens um damit, etwa in Abgrenzung zum Begriff der Platzierung, das Zusammenspiel von aktivem Handeln (ich positioniere mich) und struktureller Bedingung bzw. passivem Zugewiesenwerden (ich werde positioniert) zu verdeutlichen. Der Begriff der Selbst-Positionierung wird im Folgenden dort verwendet, wo der individuelle Akt, auch im Sinne der (ideell vollzogenen) Selbst-Verortung, besonders betont werden soll.Die Thematik formaler Staatsbürgerschaft stand anfänglich nicht im Fokus des Interesses der Studie. Die hohe Emotionalität, mit der viele Jugendliche besonders in den zweiten Interviews über das Thema sprachen, die divergierenden Haltungen gegenüber einer Einbürgerung und die Beobachtung, dass diese zwischen erstem und zweitem Befragungszeitpunkt auch wechselten, veranlassten uns jedoch dazu, diese Haltungen genauer zu untersuchen und nach ihrer jeweiligen biografischen Verortung zu fragen. Dies soll im vorliegenden Beitrag geleistet werden.

\section{Anspruch, Irrelevanz, stolzer Verzicht - drei Grundmuster in Bezug auf die Einbürgerung}

Im Rahmen unserer Analysen ${ }^{8}$ des biografischen Materials aus der Emmen-Studie lassen sich drei wesentliche Grundmuster in den Deutungen und Strategien der Jugendlichen in Bezug auf eine Einbürgerung in die Schweiz unterscheiden: erstens

einem Bundesgerichtsentscheid 2008 korrigiert werden musste. Diese besondere Situation erfordert besondere Vorsicht bei der Generalisierung der Erkenntnisse, gleichzeitig lassen sich die uns interessierenden Fragen hier gewissermassen «im Brennglas» analysieren.

6 Das qualitative Sample umfasst Jugendliche kosovarischer (10), serbischer (6), kroatischer (2), portugiesischer (8) und italienischer (8) Herkunft. Die Jugendlichen sind (bzw. waren zumindest zum ersten Befragungszeitpunkt) in der Gemeinde Emmen wohnhaft und wurden in der Schweiz geboren oder sind mit ihren Eltern im Vorschulalter zugewandert. Bei der Auswahl wurde darauf geachtet, dass in jeder Herkunftsgruppe Jugendliche beider Geschlechter und aus verschiedensten Bildungsniveaus (inkl. Gymnasium) vertreten sind.

7 Zum biografisch-narrativen Interview siehe Schütze (1983).

8 Die Analyse in der Emmen-Studie orientiert sich grundsätzlich am fallrekonstruktiven Verfahren nach Schütze (1981 und 1983) und Rosenthal (1995). Zu Methode und Methodologie der Emmen-Studie siehe auch Mey (2016). 
der Anspruch auf den Schweizer Pass, zweitens die Irrelevanz bzw. der Relevanzentzug gegenüber dem Thema Einbürgerung bzw. Staatsbürgerschaft und drittens der Verzicht auf Einbürgerung im Sinne einer selbstbewussten Definition als AusländerIn. Dabei zeigt sich, dass die unterschiedlichen Haltungen gegenüber einer Einbürgerung in engem Zusammenhang damit stehen, wie die jungen Frauen und Männer den Übergang ins Erwachsenenalter bisher erfahren haben, welche Erfahrungen sie bei der Positionierung in Bildung, Beruf und sozialen Kontexten gemacht haben und wie sie ihre gesellschaftliche Stellung in der Schweiz wahrnehmen und deuten (Mey und Rorato 2010).

Ziel der folgenden Abschnitte 2.1-2.3 ist es, die drei Grundmuster anhand ausgesuchtem biografischen Materials zu präsentieren, in den Kontext der jeweiligen biografischen Konstellationen zu stellen und auf relevante Aspekte im subjektiven Verständnis von Staatsbürgerschaft hin zu befragen. Aus Platzgründen wird auf vertiefende Einzelfalldarstellungen verzichtet, die Befunde werden in verdichteter Form präsentiert. Die Muster sind in der sozialen Realität nicht immer trennscharf und auch nicht homogen, sondern vereinen in sich vielfältige Akzentuierungen. Auch findet Wandel statt, indem Jugendliche ihre Einbürgerungsstrategien über die Zeit unter Umständen verändern. Eine eindeutige Zuordnung der Interviewten zum einen oder anderen Muster ist damit nicht immer möglich. Die im Folgenden gemachten groben Angaben zur quantitativen Verteilung der beobachteten Muster in unserem Sample dienen (lediglich) dazu, das Sample und den Wandel innerhalb des Samples besser fassbar zu machen. Rückschlüsse auf die quantitative Verteilung in der weiteren, sampleüberschreitenden sozialen Realität sind selbstverständlich nicht zulässig.

\subsection{Einbürgerung als selbstverständlicher Anspruch auf volle Mitgliedschaft und Gleichberechtigung}

Die geplante Einbürgerung erscheint hier als Teil des gesellschaftlichen Positionierungsprozesses im Übergang ins Erwachsenenalter. Der Anspruch auf die Schweizer Staatsbürgerschaft wird von den Jugendlichen mit Blick auf die Tatsache, dass man hier geboren und/oder aufgewachsen ist, mit grosser Selbstverständlichkeit geäussert. Einige Personen haben bereits einen Antrag auf Einbürgerung gestellt, sei es zusammen mit der Familie oder alleine, andere planen, dies demnächst zu tun. ${ }^{9}$ Zum ersten Befragungszeitpunkt, im Alter von rund 16 Jahren, lassen sich rund zwei Drittel der Jugendlichen diesem Muster zuordnen, zum zweiten Zeitpunkt, im Alter von rund 19 Jahren, sind es noch knapp die Hälfte, da verschiedene Personen in der Zwischenzeit zu alternativen Einbürgerungsstrategien gewechselt haben.

9 Weitere drei Personen des Samples waren bereits zum ersten Befragungszeitpunkt eingebürgert, sie vollzogen die Einbürgerung zu einem früheren Zeitpunkt zusammen mit ihren Eltern. 


\subsubsection{Biografische Konstellation}

Die jungen Frauen und Männer leben seit ihrer Geburt oder frühen Kindheit in der Schweiz. Sie sind gut vernetzt in ihrem familiären und Gleichaltrigen-Umfeld und versuchen, sich möglichst erfolgreich im Bildungssystem oder im Arbeitsmarkt zu positionieren. Je nach schulischen Voraussetzungen sind sie in eine mehr oder weniger anspruchsvolle Lehre im Dienstleistungsbereich oder in technischen Berufen eingestiegen, Ausbildungsplätze in ausländerInnenspezifischen Branchen werden nach Möglichkeit gemieden. Viele planen oder realisieren bereits Weiterbildungen, einige wenige haben nach der Volksschule den Einstieg ins Gymnasium geschafft. Die Jugendlichen gehören fast durchwegs den besonders stigmatisierten Herkunftsgruppen an (viele von ihnen sind KosovarInnen). In der Freizeit sind sie oft und gerne - und seit Abschluss der Volksschule und dem Verlust ehemaliger Klassenkameraden meist vermehrt - mit Gleichaltrigen derselben nationalen oder ethnischen Herkunft zusammen. Es bestehen vielfältige transnationale Beziehungen ins Herkunftsland, die mit zunehmendem Alter vermehrt auch unabhängig von den Eltern gelebt und gepflegt werden. Viele haben sich gegen und nach Schulende intensiv mit der eigenen Familien- und Migrationsgeschichte auseinandergesetzt und dabei zu ihrer persönlichen Spielart von (doppelter) Zugehörigkeit zu Herkunfts- und Aufnahmeland gefunden. Zum Wohnort Emmen hat man eine grundsätzlich enge emotionale Beziehung, nach wie vor leben die Familie und die wichtigsten Kolleginnen und Kollegen da, bei der zweiten Befragung kann man sich jedoch auch vorstellen, mal woanders hinzuziehen. Die Jugendlichen nehmen Differenzsetzungen und Mechanismen von Stigmatisierung und Diskriminierungen gegenüber ausländischen Personen bzw. gegenüber ihrer eigenen Herkunftsgruppe sehr differenziert wahr, beklagen sich aber kaum je über persönliche Erfahrungen von Benachteiligung aufgrund ihrer ausländischen Herkunft. Vielmehr versuchen sie, Erfahrungen von Ausschluss und Abwertung zu begegnen, indem sie vehement universalistische Werte vertreten und einfordern, als «Mensch» beurteilt zu werden. "Jeder Mensch ist gleich. Es gibt überall schlechte und gute Menschen» (Fatlum, 7.4.2006) ${ }^{10}$, lautet eine wiederholt geäusserte Argumentation.

\subsubsection{Relevante Aspekte im Verständnis von Staatsbürgerschaft}

Im geschilderten biografischen Kontext ist sowohl der Wunsch als auch der Anspruch auf eine Einbürgerung verankert. Für die jungen Frauen und Männer ist es selbstverständlich, dass eine Person, die in der Schweiz geboren oder hier aufgewachsen ist, die Kriterien für die volle Mitgliedschaft im Rahmen der Staatsbürgerschaft erfüllt. Ein junger Mann äussert sich folgendermassen: «Ich bin hier aufgewachsen, habe die Schule gemacht, habe die Lehre fast fertig. Und, ich habe nicht vor nach Kosovo zurück zu gehen, verstehst du. Und wieso sollte ich jetzt zum Beispiel

10 In Klammern wird hier und im Folgenden jeweils der Name bzw. das Pseudonym der zitierten Person sowie das Erhebungsdatum des Interviews angegeben, dem das Zitat entnommen ist. 
keinen Schweizer Pass haben»(Blerim, 5.12.2005). Ob man hier geboren wurde oder im frühen Kindesalter eingewandert ist, erscheint in den Äusserungen der Jugendlichen nicht als relevanter Unterschied (wie dies eine strenge Auslegung des ius soli, die auf den Geburtsort abhebt, implizieren würde). Der Anspruch auf Einbürgerung wird in beiden Fällen mit der gleichen Selbstverständlichkeit geäussert. "Ich bin da halt von klein an aufgewachsen und fühle mich eigentlich da geboren» (Fatlum, 4.12.2008), wird dann formuliert. Viele führen aus, dass fast ihr gesamtes soziales Umfeld hier lebe. Eine junge Frau meint schlicht: «Es ist alles hier» (Vesna, 30.12.2005). Nebst dem sozialen Umfeld und der jahrelangen Partizipation an den zentralen gesellschaftlichen Institutionen des Bildungswesens kommen dabei auch immer wieder Aspekte von (biografischer) Verbundenheit zur Sprache: «Wir sind einfach da geboren und wir fühlen uns da wohl» (Fatlum, 7.4.2006); oder: «Ich meine, ich habe alle meine Erinnerungen hier» (Blerim, 5.12.2005). Einzelne gehen noch weiter und verweisen darauf, wie gut sie und ihre Eltern «integriert» seien, indem zum Beispiel die Sprachkenntnisse oder die Berufstätigkeit hervorgehoben werden; hier lassen sich Verschiebungen hin zur Vorstellung eines «verdienten» Schweizer Passes im Kontext einer neoliberal geprägten Integrations- und Sozialpolitik beobachten (Wicker 2011). Jedoch fällt auf, dass die Jugendlichen so gut wie nie auf das Kriterium nationaler Identifikation rekurrieren in dem Sinne, dass sie beteuern würden, sich «wie Schweizer» zu fühlen. Nationale Identifikation erscheint in den Interviews zwar durchaus als Gegenstand ihrer persönlichen "Zugehörigkeitsarbeit» (Mecheril 2003, 335), und die Jugendlichen verwenden eine Fülle von Bildern und Ausdrücken, um ihre vielfältigen Beziehungen zu Herkunfts- und Aufnahmeland zu beschreiben und für sich zu definieren, aber sie erscheint nicht als Kriterium für den Zugang zu voller Mitgliedschaft und Gleichberechtigung (auch) in jenem Land, in dem sie leben. Es ist die biografische Verbundenheit, die alltägliche Lebenspraxis und mit ihr die soziale und berufliche Partizipation mit und in der Schweiz, die für die Jugendlichen entscheidend ist; man könnte in diesem Sinne auch von einem angereicherten Territorialprinzip sprechen, das hier vertreten wird.

Die Jugendlichen begründen ihren Einbürgerungsplan im Interview aber nicht nur über den Anspruch auf die Formalisierung ihrer gelebten Staatsbürgerschaft (Reichel [2011, 104] spricht in diesem Zusammenhang auch von «Statusanpassung»), sondern sie benennen auch explizit, welche konkreten Inhalte des StaatsbürgerschaftsStatus für sie besonders relevant sind. Diese haben fast durchwegs mit sozialer und rechtlicher Gleichstellung zu tun, die die Jugendlichen mit dem Status verbinden und bisher offensichtlich vermissen. Einbürgerungsgründe, die eher auf symbolischer, emotionaler Ebene liegen, wie die Ermöglichung von Gefühlen der Zugehörigkeit, werden demgegenüber selten explizit genannt (ähnlich bei Reichel 2011), und wenn, dann sind sie in den Interviews eng verbunden mit den dominanten Aspekten der Gleichstellung. "Ich meine einfach, wir sind Leute, es gibt Leute, die wirklich integriert sind und die deswegen trotzdem ein bisschen ausgeschlossen sind, weil 
sie immer noch einen anderen Pass haben»(Blerim, 23.12.2008), beschreibt ein junger Kosovare seine Wahrnehmung fehlender Gleichstellung. Und eine junge Serbin meint: "Mit dem Schweizer Pass hat man irgendwie immer einen halben Schritt Vorsprung» (Mirjana, 9.4.2009). Zwei Bereiche werden als relevanteste Aspekte der Gleichstellung angesprochen. Zum einen nennen die Jugendlichen Rechte im Zusammenhang mit der Reise- und Bleibefreiheit, die sie vermissen: Sei es im Zusammenhang mit komplizierten Visaverfahren für Reisen in die USA, in Bezug auf die Sorge, wegen des geplanten Auslandaufenthalts die Niederlassungsbewilligung zu verlieren, sei es, weil man sich die Zukunft in der Schweiz auch jenseits der Rückkehrpläne der Eltern sichern möchte - was bei uns vor allem bei jungen Frauen der Fall ist - oder sei es aus Angst, in einer Phase verstärkter Fremdenfeindlichkeit als "Ausländerin» das Land verlassen zu müssen ${ }^{11}$ : In all diesen in den Interviews genannten Fällen geht es darum, über die Einbürgerung eine rechtliche Ausstattung zu erhalten, die dem in der Schweiz verankerten und gleichzeitig transnational vernetzten Leben der jungen Menschen entspricht und ihnen ermöglicht, im Übergang ins Erwachsenenalter eigenständige Pläne und Lebensperspektiven entwickeln zu können. Zum anderen wird in den Interviews auf jene Aspekte fehlender Gleichstellung verwiesen, die nicht primär rechtlicher Natur sind, sondern sich stärker auf informelle Benachteiligungen wie die wahrgenommenen erschwerten Zugänge insbesondere zum Arbeitsmarkt für ausländische Personen und speziell für Personen stigmatisierter Herkunftsgruppen beziehen, wie diese für die Schweiz schon verschiedentlich nachgewiesen werden konnten (Fibbi et al. 2003; Fibbi et al. 2007; Imdorf 2010). «Es ist mit dem Schweizer Pass einfacher, eine Stelle zu finden» (Laura, 28.3.2009), ist ein Satz, der so oder ähnlich in den Interviews immer wieder geäussert wird. Aber auch im Freizeitbereich erhofft man sich mit dem Schweizer Pass den Wegfall von Benachteiligungen, insbesondere im Zusammenhang mit einfacheren Zutrittsbedingungen zu bestimmten Clubs und Diskotheken. ${ }^{12}$ Politische Rechte werden demgegenüber nur sehr vereinzelt explizit als relevanter Aspekt von Staatsbürgerschaft erwähnt. Dieser Aspekt der Gleichstellung scheint im Vergleich zu den anderen (noch) weniger dringlich zu sein. ${ }^{13}$ Allerdings ist mit Blick auf die Befunde zum Jugendparlament in Emmen auch festzuhalten, dass dort, wo die Möglichkeit zu politischer Partizipation offenstand, diese durchaus auch genutzt wurde (Mey und Rorato 2010).

11 "Wer weiss, vielleicht müssten ja, kommt irgendeinmal die Zeit, wo alle Ausländer raus müssen, ich weiss nicht. Ich glaube es zwar nicht ... Aber wenn es soweit kommen würde, würde ich schon gerne hier bleiben» (Mileva, 11.4.2006). So die Aussage einer bosnischen Serbin, die sie, als muslimische Jugendliche, zur Zeit der Lancierung der Minarettinitiative macht.

12 Zum Zeitpunkt der Befragung hatten verschiedene Lokale im Raum Luzern ein Zutrittsverbot für junge Männer aus Exjugoslawien verhängt.

13 Dass gerade junge Menschen ein vergleichsweise geringes Interesse an politischer Partizipation insbesondere über formale Kanäle haben, wie die Staatsbürgerschaft sie ermöglicht, beschreibt u. a. Walther (2011). 
So selbstverständlich für die jungen Frauen und Männer ihr Anspruch auf die formale Staatsbürgerschaft ist, so irritiert, verärgert oder enttäuscht wird auf Anzeichen eines erschwerten Erwerbs des Schweizer Passes reagiert. Lange Wartezeiten und hohe Kosten für die Einbürgerung werden nicht nur per se als störend wahrgenommen, dies insbesondere angesichts des noch geringen Einkommens während der Ausbildung, sondern auch als Signal, dass die Einbürgerung alles andere als selbstverständlich ist. "Ich zahle den Pass schon, die Arbeit von den Angestellten, die sie dafür haben", differenziert ein junger Befragter, «aber wieso sollte ich dafür ein- oder zweitausend Franken bezahlen. Das verstehe ich nicht» (Blerim, 23.12.2008). Einige Jugendliche nennen hohe Gebühren oder lange Wartezeiten denn auch explizit als Anlass, von ihrem Einbürgerungsvorhaben ganz oder zumindest vorerst abzusehen (vgl. unten, Relevanzentzug).

\subsubsection{Zusammenfassung / Typisierung}

Die faktisch realisierte und alltäglich gelebte Partizipation an und in der Schweizer Gesellschaft - die gelebte Staatsbürgerschaft - soll durch die volle Mitgliedschaft und politisch-rechtliche Gleichstellung im Rahmen der formalen Staatsbürgerschaft anerkannt und vervollständigt werden. Dabei gilt es, das in der Schweiz verankerte und transnational vernetzte Leben abzusichern und die bisherigen Positionierungserfolge in Beruf und Bildung zu konsolidieren (vgl. auch Fibbi et al. 2007). Die Einbürgerung ist Teil der gesellschaftlichen Positionierung im Übergang ins Erwachsenenalter in der Schweiz. Als solche ist sie meistens eingebettet in ein familiales Projekt, in einigen Fällen ist sie umgekehrt gerade Basis von Lebensentwürfen jenseits der elterlichen Pläne - in unserem Sample betrifft dies ausschliesslich weibliche Jugendliche. Grundsätzlich wird ein Verständnis von nationalstaatlicher Zugehörigkeit vertreten und eingefordert, das Staatsbürgerschaft nicht an ethnische Herkunft und auch nicht an nationale Identifikation, sondern an gelebte Partizipation und Zugehörigkeit (und allenfalls Leistung) bindet. Eingebettet in einen Lebensentwurf, der auf die Erreichbarkeit bzw. Erwerbbarkeit gesellschaftlicher Positionen in allen Bereichen baut, birgt dieser Einbürgerungswunsch ein besonderes Verletzungspotenzial; Enttäuschungen und negative Signale in Bezug auf die Zugänglichkeit des Status als vollwertiges und gleichberechtigtes Gesellschaftsmitglied können so zu veränderten Zugehörigkeits- und damit auch Einbürgerungsstrategien führen.

\subsection{Fehlende oder entzogene Relevanz des Themas Einbürgerung}

In diesem Typus besteht kein Plan für eine Einbürgerung in die Schweiz, weil man dazu schlicht keinen Anlass (mehr) sieht. Dem Thema Einbürgerung wird im biografischen Entwurf keine oder eine stark untergeordnete Rolle beigemessen. Das Muster findet sich in zwei Varianten: a) bei Jugendlichen, die bisher noch nie ernsthaft an eine Einbürgerung gedacht haben oder b) bei Jugendlichen, die einen ursprünglichen Einbürgerungsplan fallengelassen und dem Thema bewusst die 
Relevanz entzogen haben. Auch wenn sich in den beiden Varianten also je andere biografische Konstellationen verbergen, sind die wesentlichen Aspekte in der Haltung gegenüber der Staatsbürgerschaft identisch. In der ersten Befragung weisen vereinzelte Jugendliche, in der zweiten gut ein Viertel der Interviewten das Muster fehlender bzw. entzogener Relevanz auf. ${ }^{14}$

\subsubsection{Biografische Konstellation}

\section{(Noch) keine Relevanz}

Hier finden sich junge Frauen oder Männer, die bereits in der Volksschule die besser qualifizierten Bildungsgänge besucht und im Anschluss rasch eine Lehre mit erhöhtem Anforderungsprofil im Dienstleistungssektor gefunden haben (kaufmännischer Bereich, Informatik) oder ins Gymnasium eingetreten sind. Sie gehören den am wenigsten stigmatisierten Herkunftsgruppen unseres Samples an (meist ItalienerInnen) und fühlen sich in der Schweiz grundsätzlich wohl und anerkannt. Dazu gehört auch, dass die Jugendlichen wahrnehmen, wie ihr Herkunftsland im Unterschied zu den Herkunftsländern anderer ausländischer Jugendlicher ein positives Image besitzt. «Das ist ja nicht ein blödes Land oder so», meint etwa ein junger Italiener, «das gefällt ja noch vielen» (Giancarlo, 18.9.2007). Zu Emmen hat man eine gute, aber keine besonders enge Verbindung. Der Freundeskreis variiert in Bezug auf die nationale Herkunft, bei einigen ist er fast ausschliesslich italienisch, bei anderen mehrheitlich schweizerisch. Es bestehen verschiedentlich Pläne für Auslandreisen und -aufenthalte.

\section{Relevanzentzug}

Auch die Jugendlichen, die der Einbürgerung die Relevanz entzogen haben, sind daran, sich möglichst erfolgreich in Bildung oder Beruf zu positionieren. Sie sind als ehemals Einbürgerungswillige - jedoch vorwiegend kosovarischer, kroatischer oder serbischer Herkunft, und sie haben im Zusammenhang mit ihrem einstigen Einbürgerungswunsch Enttäuschungen erlebt oder negative Signale erhalten, über die sie im zweiten Interview berichten. Den Rückzug vom einstigen Einbürgerungsvorhaben stellen sie selber primär in diesen Kontext. Ausserdem ist erkennbar, dass der Relevanzentzug auf einen Zeitpunkt fällt, zu dem die Jugendlichen erste ihnen wichtige Erfolge bei der beruflichen Positionierung gemeistert haben. Diese Erfolge, so unsere Interpretation, stärken und ermuntern die Jugendlichen darin, sich nach der erfahrenen Enttäuschung beim Bemühen um politisch-rechtliche Gleichstellung künftig verstärkt auf die ökonomische Sphäre zu konzentrieren.

14 Mit eingerechnet sind hier jene drei Personen, deren Entscheid zur Einbürgerung inzwischen abgelehnt wurde. Ihre dominante Strategie im Umgang mit der Ablehnung besteht zumindest im Versuch eines Relevanzentzugs - verbunden mit mehr oder weniger ausgeprägten Ohnmachtsgefühlen. Auf die spezifische Situation von Jugendlichen mit einem ablehnenden Entscheid muss andernorts differenziert eingegangen werden. 


\subsubsection{Relevante Aspekte im Verständnis von Staatsbürgerschaft}

\section{(Noch) keine Relevanz}

«Mir geht es eigentlich gut so, wie es jetzt ist. Ich werde ja akzeptiert von allen, auch wenn ich jetzt nicht eingebürgert bin, und ja, das ist für mich eigentlich kein Problem momentan, und sobald das kein Problem ist, dann, ist das ok so» (Frederica, 21.8.2009), sagt etwa eine junge Italienerin mit gutem Bildungsrucksack: Für sie schien die Einbürgerung bisher noch nie wichtig oder dringlich. Ihr Argument, von allen akzeptiert zu werden, weist darauf hin, dass sie der Staatsbürgerschaft zumindest ein gewisses Potenzial zuschreibt, gesellschaftliches Ansehen und Akzeptanz zu verleihen - nur ist dieser Aspekt für sie persönlich irrelevant, da sie sich sowieso schon anerkannt fühlt und ihre Ziele bisher ohne nennenswerte Hindernisse verfolgen konnte. Ganz ähnlich klingt es bei der portugiesischen Jugendlichen (die mit der Ausbildung zur Kauffrau als einzige Portugiesin des Samples eine Lehre mit erhöhtem Anforderungsprofil macht): «Ich habe jetzt noch nie einen Nachteil gehabt oder sagen müssen, ja, warum brauche ich das jetzt» (Tereza, 7.8.2009). Bei einigen männlichen Jugendlichen mit dem Muster Irrelevanz wird die fehlende Notwendigkeit ergänzt und akzentuiert dadurch, dass sie aufgrund der allgemeinen Wehrdienstpflicht mit einer Einbürgerung nicht nur keine Vorteile, sondern auch Nachteile verbinden.

\section{Relevanzentzug}

Auch hier wird auf die fehlende Notwendigkeit der Einbürgerung verwiesen, auch wenn die entsprechenden Formulierungen zum Teil noch etwas zögerlich klingen, wie etwa in folgenden Worten einer bildungsbewussten jungen Kosovarin, die ihre schon weit vorangeschrittenen Einbürgerungspläne - sie hatte sich bei der Gemeinde bereits die Unterlagen besorgt - sistiert hat und dies mit den antizipierten Hürden begründet («in Emmen ist es sowieso sehr schwierig»): «Eigentlich habe ich es nicht so so nötig. Ich meine ich habe einen Pass mit dem ich überall hinreisen kann ... und es ist mir eigentlich nicht so so wichtig» (Ardita, 20.11.2008). Auch ein junger Kroate, der nach Abschluss seiner qualifizierten Lehre mit dem Antritt einer neuen Stelle auch die zweite Schwelle auf seinem Bildungsweg gemeistert hat, sagt, dass eine Einbürgerung für ihn nicht (mehr) dringlich sei. Dabei kommt in seinen Worten vor allem Unverständnis und Ärger angesichts langer Wartezeiten zum Ausdruck: «Sie (die Behörde, E. M.) haben einen Brief geschickt, dass ich warten soll. Ja was heisst warten? Soll ich da jetzt zehn Jahre warten auf den Schweizer Pass oder was? Nein, da bin ich nicht drauf angewiesen» (Andrej, 7.10.2008). Aspekte der Gleichstellung, die man früher noch mit der Staatsbürgerschaft verbunden und als relevant erachtet hatte, werden nun als weniger schwerwiegend bewertet. Wichtige Reiserechte hat man inzwischen ohnehin erhalten ${ }^{15}$, und die bisher erreichten beruflichen Fortschritte

15 Dies betrifft vor allem die Jugendlichen kosovarischer Herkunft, die erst mit der Unabhängigkeit des Kosovo anfangs 2008 (und damit zwischen erstem und zweitem Befragungszeitpunkt) die Möglichkeit erhielten, sich ohne Visa im Schengen-Raum zu bewegen. 
ermutigen, auch den möglichen Wegfall informeller Benachteiligung beim Zugang zu Bildung und Beruf als nicht (mehr) bedeutsam zu definieren.

Auffallend ist eine generelle Ablehnung der Bedeutung ethnisch geprägter Kategorien in den Erzählungen dieser Jugendlichen. Dies zeigt sich an expliziten Aussagen («Nationalität ist für mich nicht so wichtig» [Milan, 3.10.2009]), aber auch an der Art und Weise, wie biografische Erfahrungen in den verschiedensten Bereichen erzählt werden, ob und inwieweit sie national bzw. ethnisch konnotiert werden oder nicht. Entsprechend wird auch der Rückzug vom Einbürgerungsplan in keiner Weise im Rückgriff auf ethnische Kategorien beschrieben oder begründet - dies im Unterschied zu den Jugendlichen, auf die weiter unten im Rahmen des dritten Musters eingegangen wird.

\subsubsection{Zusammenfassung / Typisierung}

Die jungen Frauen und Männer bilden sich in der Regel in besser qualifizierten Berufen aus und blicken zuversichtlich in ihre berufliche Zukunft. Unter den Jugendlichen, die der Einbürgerung die Relevanz entzogen haben, finden sich allerdings auch einzelne in (noch) weniger privilegierten beruflichen Positionen. Ob und inwieweit die Staatsbürgerschaft allenfalls das Potenzial nicht nur zu rechtlicher, sondern auch zu sozialer Gleichstellung im Sinne des Wegfalls informeller Benachteiligung beinhalten würde, interessiert nicht oder nicht mehr, weil man darauf sowieso nicht angewiesen ist oder zu sein hofft. Das zentrale Motiv ist Autonomie - was die jungen Frauen und Männer dafür brauchen und suchen, ist eine gute Positionierung in Bildung und Beruf. Der Rückzug von den bisherigen Einbürgerungsplänen wird in keiner Weise im Rückgriff auf ethnische Aspekte begründet. Im Kontext ethnisierter und politisierter Einbürgerungsdiskurse lässt sich der Rückzug als Versuch deuten, die Relevanz ethnischer Kategorien in der eigenen Biografie bewusst zu relativieren und zu überwinden.

\subsection{Selbstbewusster Verzicht auf Einbürgerung - Positionierung als Ausländerln}

Im Rahmen dieses Grundmusters wird von einer Einbürgerung bewusst abgesehen. Der Verzicht auf eine Einbürgerung kommt einem Statement gleich, mit dem man der Liebe zum Herkunftsland, in einer zugespitzten Variante der Selbstverortung als Ausländerin bzw. Ausländer in der Schweiz, Ausdruck verleihen will. Knapp ein Viertel der Jugendlichen unseres Samples lässt sich diesem Muster zuordnen. Verschiedene von ihnen äusserten beim ersten Interview eine Tendenz zu dieser Haltung, waren aber noch unentschieden; in der zweiten Befragung hat sich ihr Entscheid zum selbstbewussten Verzicht auf die Einbürgerung klar verfestigt.

\subsubsection{Biografische Konstellation}

Die jungen Frauen und Männer lassen sich zum Teil fast der dritten Generation zuzählen: Ihre Eltern sind selber meist schon in sehr jungen Jahren auf der Suche 
nach Arbeit in die Schweiz gekommen und haben sich oft erst hier kennengelernt. Es handelt sich vorwiegend um Portugiesinnen und Portugiesen. Arbeit fand sich vor allem im Bau, in der Pflege oder in der Reinigung, wo die Eltern seither tätig sind. Schon früh nehmen die Kinder wahr, wie hart ihre Eltern arbeiten und wie knapp das Geld trotzdem oft ist. Die Kinder selber werden aufgrund ihrer (ebenfalls wenig privilegierten) schulischen Voraussetzungen bei der ersten schulischen Selektion in der Regel dem Schulniveau mit niedrigem Anforderungsniveau zugeteilt. Nach der Schule finden sie über verschiedene Mechanismen und Stationen ${ }^{16}$ zumeist in denselben ausländerInnenspezifischen Branchen einen beruflichen Ausbildungsplatz, in denen auch ihre Eltern arbeiten (v. a. Bau oder Pflege), meist entgegen ursprünglichen Plänen und Wünschen. Differenzsetzungen und unterschiedliche Positionen zwischen «den Schweizern» und «den Ausländern» in der Schweizer Gesellschaft werden hier deutlich wahrgenommen und im Interview auch thematisiert. Es sind jene Jugendlichen, die im Rahmen der ersten schulischen Selektion beobachtet und untereinander diskutiert haben, dass es in der Realschule besonders viele ausländische Kinder hat, während die höheren Bildungsgänge vornehmlich von SchweizerInnen besucht werden, und die später, im Rahmen der Berufssuche, lapidar feststellen: «Die Baustellen sind ja voller Ausländer» (Carlos, 6.2.2009). Sich selber definieren die Jugendlichen dabei selbstverständlich als Ausländerin bzw. als Ausländer. Die Gleichaltrigenkontakte umfassen Personen derselben Herkunft, allenfalls «andere Latinos», wie es eine junge Frau portugiesischer Herkunft ausdrückt - ein Muster, das sich auch hier nach Abschluss der Volksschule verstärkt und verfestigt hat. Man liebt sein Herkunftsland bzw. das Herkunftsland der Eltern und ist stolz darauf, ohne zwingend auch relevante persönliche Kontakte zu haben. Einige sind nach der Schule in einen Tanz- oder Folklorekurs eingetreten. Die lokale Zugehörigkeit zu Emmen ist ausgeprägt, fast alle wichtigen Kolleginnen und Kollegen sind hier. Sollte man in der Schweiz bleiben, möchte man nie von Emmen wegziehen. Allerdings kann man sich auch gut vorstellen, später mal im Herkunftsland der Eltern zu leben, und /oder man spielt mit dem Gedanken, zu Verwandten in ein Drittland zu ziehen. Es lässt sich hier von einer spezifischen Kombination aus lokaler und transnationaler Zugehörigkeit sprechen, die auch in anderen Untersuchungen thematisiert wird (Schiffauer 2004; Dahinden 2010; Yildiz 2010).

\subsubsection{Relevante Aspekte im Verständnis von Staatsbürgerschaft}

In dieser Konstellation ist es für die jungen Frauen und Männer so gut wie unvorstellbar, einen Schweizer Pass zu erwerben. Zwar erwähnen sie immer wieder, dass ihnen mögliche Vorteile der Einbürgerung bewusst sind: «Für die Jobsuche wäre es schon gut” (Ilir, 26.2.2009) ist eine Äusserung, die in den Interviews in vergleichbarem Wortlaut immer wieder auftaucht. Dabei erwähnen die Jugendlichen auch, dass ihre Eltern ihnen die Einbürgerung aufgrund der damit verbundenen Vorteile

16 Dazu gehören auch Mentoringprojekte und Brückenangebote, vgl. Mey (2015). 
nahegelegt hätten und sich für ihre Kinder die Schweizer Staatsbürgerschaft wünschen. Ein junger Mann: «Meine Mutter hat es mir auch schon gesagt, wieso machst du es nicht oder so. Das ist halt wieder der Stolz den ich im Kopf habe, dass ich ... wegen dem Portugiese sein ... dass ich nicht etwas anderes sein will oder so (lacht). (...) Auch bei der Jobsuche hat man vielleicht ein bisschen mehr Vorteile. Aber das ist mir irgendwie egal, entweder akzeptieren sie mich wie ich bin oder ... dann muss ich mich auch nicht einbürgern» (Jorge, 3.3.2009). Ein anderer Jugendlicher hält lapidar fest: «Ich bin stolzer Ausländer, ich sag es so» (Carlos, 6.2.2009). Der Verzicht auf die Schweizer Staatsbürgerschaft erscheint hier als bewusstes Statement, als stolz-widerständige Positionierung als AusländerIn, mit der sich die Jugendlichen nicht nur dem Assimilationsgebot im Rahmen dominanter Einbürgerungsdiskurse, sondern auch den (Anpassungs-)Wünschen ihrer Eltern, die für die Kinder den Schweizer Pass vorgesehen hätten, entziehen und widersetzen. Bemerkenswert ist, dass die Jugendlichen zwar mit den aktuellen Vorstellungen ihrer Eltern brechen, jedoch gerade damit deren Stellung als im Wohnland politisch-rechtlich nicht gleichgestellte Mitglieder der Gesellschaft reproduzieren. Angesichts dessen, dass die Jugendlichen in den Interviews oft identische Formulierungen verwenden, wenn sie über ihre eigenen und die Vorstellungen ihrer Eltern sprechen, gehen wir davon aus, dass ihre Haltung stark jugendkulturell geprägt ist, das heisst die Vermittlung über kollektive Diskurse unter Jugendlichen eine wichtige Rolle spielt (Bohnsack 1999).

Dabei nutzen und spiegeln die Jugendlichen just jenes stark herkunftsbezogene, ethnisierte Verständnis von Staatsbürgerschaft, das sie in den dominanten Einbürgerungsdiskursen vermittelt bekommen. Liebe, Verbundenheit und Stolz auf das eigene Herkunftsland (bei einigen verbunden mit Stolz auf die eigenen Eltern, die ihr Leben «als Ausländer» gemeistert haben) verunmöglichen gewissermassen die Übernahme einer anderen Staatsbürgerschaft: «Einfach mein Heimatland ist mein Heimatland, was will ich machen. Ich bin Portugiese, ich bin so auf die Welt gekommen und jetzt bin ich halt so und das bleibt auch so. Deswegen bleibe ich immer stolz auf mein eigenes Heimatland»(Carlos, 6.2.2000). Oder ein junger Italiener: "Ich kann es mir einfach nicht vorstellen, überhaupt nicht ... Ich bin als Italiener geboren» (Alessio, 7.3.2007). Auffallend sind hier die Unterschiede zu den Jugendlichen des ersten Musters (Anspruch): Anders als jene - die in den Interviews durchaus auch von ihrer Liebe zur Heimat sprechen - verbinden diese Jugendlichen hier ihre Liebe zum Herkunftsland explizit auch mit Gefühlen von Stolz. Dass sie Stolz überhaupt empfinden und auch äussern können, sehen wir dabei nicht zuletzt im positiveren Image ihres Herkunftslandes begründet (Italien, Portugal im Gegensatz zu Ländern des ehemaligen Jugoslawien): Die Selbst-Positionierung als Ausländerin oder als Ausländer kann hier symbolisch aufgewertet und positiv konnotiert werden.

Bei den meisten Jugendlichen hat sich das Muster des stolzen Verzichts zwischen erstem und zweitem Befragungszeitpunkt verfestigt. Auch wenn sie beim ersten Interview mit Blick auf allfällige Benachteiligungen bei der Lehrstellensuche noch 
eher zögerten, äussern sie sich jetzt deutlich entschiedener gegen die Einbürgerung. «Ich habe ja jetzt eine Stelle» (Raquel, 5.11.2008), heisst es dazu unter anderem. Die Verfestigung des Musters dürfte nicht nur auf die abgenommene Dringlichkeit des Schweizer Passes zurückzuführen sein, sondern auch auf die Tatsache der Positionierung in einer ausländerInnenspezifischen Branche, die der Selbst-Positionierung als Ausländerin und Ausländer zusätzlich Vorschub leistet.

\subsubsection{Zusammenfassung / Typisierung}

Der Verzicht auf die Schweizer Staatsbürgerschaft hat hier den Stellenwert eines bewussten Statements, einer stolz-widerständigen Positionierung als AusländerIn, bei der sich Liebe zum und Verbundenheit mit dem Herkunftsland verbinden mit der Wahrnehmung, in der Schweiz einen Platz (nur) als arbeitende Ausländerin bzw. arbeitender Ausländer innezuhaben. Es würde zu kurz greifen, den Verzicht auf die Einbürgerung als simple Reaktion auf erfahrene Diskriminierung oder Stigmatisierung zu deuten. Wir interpretieren ihn als Ausdruck einer spezifischen Selbst-Positionierung, deren Genese sich in den untersuchten Biografien nachvollziehen lässt. Diese Positionierung ist das Resultat eines langjährigen, wechselseitigen Zusammenspiels von Zuschreibungen von aussen, Wahrnehmungen geringer Chancen u. a. bei schulischen Übertritten sowie subjektiven Deutungen, die im Rahmen jugendkultureller Diskurse vermittelt werden. Aus adoleszenztheoretischer Sicht ist erwähnenswert, dass sich die Jugendlichen mit ihrem stolzen Verzicht oft auch von den (Einbürgerungs-)Erwartungen ihrer Eltern emanzipieren. Mit der beruflichen Positionierung im Rahmen der Lehrstellensuche verfestigt sich der Entschluss gegen die Einbürgerung, dies auch, weil sich damit ein ehemals dringlicher Grund (Sicherung des Zugangs zu einer Lehrstelle) erübrigt hat. Der Verzicht wird mit der Liebe zum Herkunftsland und unter Rückgriff auf ein stark ethnisierendes, herkunftsbezogenes Konzept von Staatsbürgerschaft bzw. nationaler Zugehörigkeit begründet. Die im öffentlich-medialen Kontext erfahrene Politisierung von Fragen nationaler Identifikation und Zugehörigkeit wird also nicht abgelehnt oder ignoriert, sondern übernommen, reproduziert und für die selbstbewusste Positionierung als AusländerIn nutzbar gemacht.

\section{Diskussion}

In der Adoleszenz beginnen Jugendliche, eigene Lebensentwürfe zu entwickeln und sich dabei eigenständig in Bezug auf berufliche, soziale und politische Kontexte zu positionieren. Gerade in Migrationsgesellschaften ist die Aushandlung sozialer Zugehörigkeiten - zu denen auch nationalstaatlich gefasste Zugehörigkeit zu zählen ist - ein wesentlicher Aspekt der adoleszenten Selbst-Positionierung und gerahmt durch gesellschaftlich dominante Konzeptionen von Zugehörigkeit und Nicht- 
Zugehörigkeit (Mecheril und Hoffarth 2006). Söhne und Töchter aus zugewanderten Familien sind in spezifischer Weise vom restriktiven Zugang zur Schweizer Staatsbürgerschaft und einem stark ethnisch geprägten Verständnis nationaler Zugehörigkeit betroffen. Wie ihre einheimischen Gleichaltrigen hier geboren, aufgewachsen und ausgebildet, wird ihnen aus politisch-rechtlicher Sicht volle Mitgliedschaft und Gleichberechtigung nicht automatisch zugestanden. Die vorangehend präsentierten Muster in der Haltung gegenüber einer Einbürgerung zeigen, dass die Jugendlichen unterschiedlich auf diese Ausgangskonstellation reagieren. In den Analysen wurde herausgearbeitet, wie sich individuelle Einbürgerungsentscheide im Zusammenspiel von politisch-rechtlichen Rahmenbedingungen, der spezifischen Stellung im sozialen Raum und den Deutungen und Strategien der Jugendlichen herausbilden. Dabei wird auch erkennbar, in welcher Weise Nationalität und Stellung in Bildung und Beruf - als Faktoren, die die quantitative Einbürgerungsforschung als bedeutsam identifiziert - im biografischen Zusammenhang wirksam werden. Im Folgenden werden die wichtigsten Aspekte unserer Befunde nochmals benannt und im vergleichenden Blick auf die drei identifizierten Muster diskutiert.

Nationalität und Einbürgerung: Auffallend ist die starke nationale Prägung der identifizierten Muster. Die Einbürgerungsstrategien stimmen weitgehend, wenn auch nicht durchgängig, mit bestimmten Herkunftsnationen überein: In Typus 1 finden sich vorwiegend KosovarInnen, SerbInnen und KroatInnen, in Typus 2 ItalienerInnen (bzw. Personen exjugoslawischer Herkunft in Typus 2b), in Typus 3 vorwiegend PortugiesInnen. Unsere Befunde spiegeln damit statistische Befunde zum Zusammenhang von Nationalität und (erfolgter) Einbürgerung, sowohl in Bezug auf die Relevanz von Nationalität als auch in Bezug auf die höheren Einbürgerungsraten bei Angehörigen aus Nicht-EU-Ländern (vgl. Wanner und Steiner 2012). Dennoch ist festzuhalten, dass Nationalität und Typus nicht immer deckungsgleich sind, sondern dass es - aussagekräftige - Ausnahmen gibt. Diese unterstreichen die hohe Bedeutung, die der Stellung in Bildung und Beruf für die Einbürgerungsstrategie zukommt. In unseren Analysen sind es erstens die unterschiedlich starken Stigmatisierungen, denen die einzelnen Herkunftsgruppen in der Schweiz ausgesetzt sind, und zweitens die je nach Herkunftsgruppe (und Migrationsgrund) anderen Muster der Positionierung im Arbeitsmarkt, die sich als entscheidend für die hohe Übereinstimmung zwischen Nationalität und Einbürgerungsstrategie bzw. -wunsch erweisen.

Einbürgerungsstrategien im Kontext von sozialer Benachteiligung und sozialem Ausschluss: Die Analysen geben Einblick in die vielfältigen und differenzierten Zusammenhänge zwischen den Erfahrungen von sozialer Benachteiligung, Diskriminierung und Stigmatisierung einerseits, über die sich den Jugendlichen ihre Stellung im sozialen Raum vermittelt, und den gewählten Einbürgerungsstrategien andererseits. Im Rahmen des ersten Typus lässt sich erkennen, wie sehr sich die vorwiegend kosovarischen und serbischen Jugendlichen des negativen Images ihrer Herkunft bewusst sind. Es drängt sie unter diesen Bedingungen in besonderem Masse, ihre 
Stellung, die sie sich über den Bildungsweg erkämpfen, durch die Formalisierung ihrer Mitgliedschaft zu konsolidieren und abzusichern; ihr Projekt der sozialen Mobilität verbindet sich mit einem Projekt der Anerkennung und Gleichstellung im nationalstaatlichen Kontext. Je erfolgreicher sich diese Jugendlichen positionieren, desto dringlicher formulieren sie den Wunsch nach Einbürgerung. Allerdings gilt dieser Zusammenhang nicht uneingeschränkt: Wie gezeigt können Erfolge bei der Positionierung in Bildung und Beruf auch ermutigen, vom Einbürgerungsvorhaben abzulassen (Wechsel zu Typus 2), wenn dessen Realisierung auf Widerstände stösst oder sich als nicht gesichert erweist. Das Projekt der Mobilität wird dann verstärkt zu einem Projekt der persönlichen Autonomie, das sich jenseits politisch-rechtlicher Gleichstellung auf die ökonomische Sphäre fokussiert. Nochmals anders gestaltet sich das Verhältnis zwischen marginalisierter Stellung und Haltung gegenüber der Schweizer Staatsbürgerschaft in der dritten Gruppe. Die marginalisierte Stellung ist hier nicht primär durch das negative Image der Herkunftsgruppe bestimmt, sondern durch die Positionierung von Eltern und Kindern in ausländerInnenspezifischen Branchen des Arbeitsmarktes, wie sie für die portugiesische Arbeitsmigration besonders typisch ist. Hier sind es, wie gezeigt, vielfältige Mechanismen von wechselseitigem Selbst- und Fremdausschluss, über die soziale Benachteiligung schliesslich zu einer Selbst-Positionierung als Ausländerin bzw. Ausländer führt, die in Verbindung mit der Liebe zum Herkunftsland als unvereinbar mit einer Einbürgerung angesehen wird. Staatsbürgerschaft als Modus der Reproduktion sozialer Ungleichheit: Unsere Befunde machen das Potenzial sichtbar, das individuelle Einbürgerungsstrategien für die Reproduktion marginalisierter Stellungen haben. Es sind zwei Konstellationen, die in diesem Zusammenhang besonders zu thematisieren sind. Die augenfälligste betrifft den dritten Typus: Dort, wo sich die Jugendlichen, wie beschrieben, als stolze AusländerInnen positionieren und auf ihre (auch global verwertbare) Arbeitskraft setzen, entziehen sie sich zwar der dominanten Assimilationsanforderung im nationalen Rahmen, reproduzieren aber auch in auffallender Weise Habitus und Status des Modells des Gastarbeiters, das ihre Eltern seinerzeit als «Pioniere der Globalisierung» (Yildiz und Hill 2015, 11) in die Schweiz geführt hatte. Die tendenziell marginalisierte Stellung ihrer Eltern überträgt sich dabei nicht nur über die ökonomische, sondern auch über die politische Sphäre. Der Zugang zum (nationalen) Arbeitsmarkt gestaltet sich unter anderem aufgrund informeller Benachteiligung von NichtBürgerinnen und Bürgern als schwierig, die dann erzielte Stellung im Arbeitsmarkt (in ausländerInnenspezifischen Positionen) begünstigt ihrerseits wieder den Verzicht auf die Einbürgerung: Staatsbürgerschaft kann als Kapital angesehen werden, das sich verwerten lässt oder nicht, und fungiert als Modus der Reproduktion sozialer Ungleichheit (Bauder 2008). Auch wenn transnationale Perspektiven als Alternative offenstehen, täuscht dies nicht darüber hinweg, dass sich der (Ungleichheits-)Status auch im globalisierten Arbeitsmarkt kaum überwinden lässt. Die andere Konstellation, der das Risiko einer Verfestigung und Reproduktion marginalisierter Stellungen 
anhaftet, betrifft jene zunächst einbürgerungswilligen Jugendlichen aus besonders stigmatisierten Herkunftsgruppen, die der Einbürgerung als Reaktion auf die erschwerte Zugänglichkeit die Relevanz entziehen, auch wenn ihre Positionierung im Arbeitsmarkt noch nicht nachhaltig ist (Typus 2b). Besonders hier werden weitere Beobachtungen zeigen müssen, ob und inwieweit die fehlende politisch-rechtliche Gleichstellung später in allenfalls prekäre Lebenssituationen führt.

Staatsbürgerschaft im Wandel: Werden die Einbürgerungsstrategien im Hinblick auf das ihnen zugrunde liegende Verständnis von Staatsbürgerschaft betrachtet, wird sichtbar, wie die Jugendlichen mit ihren selbstbewussten Positionierungen und Relevanzsetzungen den gesellschaftlichen Bedeutungswandel von Staatsbürgerschaft zum Ausdruck bringen und ihrerseits mitkonstituieren - und sich damit an den «sozialen Auseinandersetzungen um das knappe Gut der Staatsbürgerschaft» (Mackert 2000, 644) beteiligen. Im Rahmen des ersten Musters tun sie dies, indem sie konsequent ein Verständnis von Staatsbürgerschaft vertreten, das nicht auf nationale Identifikation, sondern auf gelebte Partizipation gründet und auf rechtliche Inhalte fokussiert. Die Entkopplung von Rechten und Identitäten, die Soysal (1994) in ihrer Studie "Limits of Citizenship" beschrieben hatte, lässt sich also auch hier beobachten. Jenseits ihrer unter Umständen vielfältigen sozialen Verflechtungen ins Herkunftsland und ihrer persönlichen Empfindung von (auch doppelter) Zugehörigkeit fordern die jungen Menschen grundsätzlich universale Kriterien des Zugangs zur Staatsbürgerschaft und Gleichberechtigung in jenem Land, in dem sie leben. Im Rahmen des zweiten und des dritten Musters wird mit dem Desinteresse an der Einbürgerung auch deren abnehmende Bedeutung - die sich unter anderem im Recht auf Mobilität im europäischen Raum ausdrückt - gespiegelt. Werden ethnisierte nationale Zugehörigkeitskonstrukte im einen Fall relativiert und zu überwinden versucht, wird im anderen Fall ein betont ethnisches Konzept von Staatsbürgerschaft vertreten, dem dann allerdings keine andere Bedeutung mehr beigemessen wird als diese (nur) symbolische.

Adoleszenzspezifische Aspekte: Während davon auszugehen ist, dass Einbürgerungen im Kindesalter immer familial gerahmt sind, erscheinen die beschriebenen Einbürgerungsstrategien in der hier untersuchten Altersspanne manchmal ebenfalls (noch) eingebettet in familiäre Projekte, manchmal jedoch - und über den Befragungszeitraum hinweg zunehmend - gerade als Basis für eigenständige Lebensentwürfe, die sich von den Plänen oder auch Erwartungen der Eltern mehr oder weniger bewusst absetzen. Dabei gibt es im Material auch Hinweise auf die Bedeutung jugendkultureller Diskurse bei der Herausbildung und Verfestigung bestimmter Haltungen zur Einbürgerung. Diese Punkte wären nicht zuletzt auf die sich abzeichnende geschlechterspezifische Prägung hin in weiteren Analysen zu vertiefen. ${ }^{17}$

17 Generell ist die Bedeutung des Faktors Geschlecht für die Herausbildung der Einbürgerungsstrategien in unseren Analysen noch wenig herausgearbeitet worden. Festhalten lässt sich immerhin, dass in jedem Typus zu vergleichbaren Anteilen sowohl Frauen als auch Männer vertreten sind. 


\section{$4 \quad$ Schluss}

In diesem Beitrag sind anhand des bisher vorliegenden biografischen Materials aus einer qualitativen Langzeitstudie zur gesellschaftlichen Positionierung von Jugendlichen der zweiten Ausländergeneration drei Grundmuster in den Haltungen gegenüber einer Einbürgerung in die Schweiz identifiziert worden. Dabei wurde dargelegt, wie die Einbürgerungsstrategien eng mit den (Selbst-)Positionierungen der Jugendlichen verbunden sind und in welcher Weise spezifische Erfahrungen von sozialer Benachteiligung und sozialem Ausschluss bei deren Herausbildung wirksam werden. Unsere bisherigen Befunde liefern Anhaltspunkte dafür, dass sich wenig privilegierte und marginalisierte Positionen im sozialen Raum auch über den Zugang zur Staatsbürgerschaft und über individuelle (auch widerständige) Einbürgerungsstrategien reproduzieren. Die dritte Befragung der Jugendlichen in Emmen, die aktuell in Vorbereitung ist, wird zeigen müssen, ob aktuell vorhandene Einbürgerungswünsche überhaupt realisiert werden (können), und ob und unter welchen Bedingungen eine fehlende Einbürgerung in eine Verfestigung sozialer Marginalisierung führt. Die hier präsentierten Befunde verstehen sich damit als Zwischenfazit. Es gilt, sie im Rahmen der längerfristigen Beobachtung unseres Samples, aber auch im Rahmen weiterer Untersuchungen, die mit anderen Samples und komparativen Designs arbeiten, zu erweitern, zu differenzieren und zu erhärten.

\section{$5 \quad$ Literaturverzeichnis}

Achermann, Christin und Stefanie Gass. 2003. Staatsbürgerschaft und soziale Schliessung. Eine rechtsethnologische Sicht auf die Einbürgerungspraxis der Stadt Basel. Zürich: Seismo Verlag.

Bauder, Harald. 2008. Citizenship as capital: the distinction of migrant labor. Alternatives 33: 315-333.

Bloemraad, Irene, Anna Korteweg und Gökçe Yurdakul. 2008. Citizenship and immigration: Multiculturalism, assimilation, and challenges to the Nation-State. Annual Review of Sociology 34: 153-179.

Bohnsack, Ralf. 1999. Rekonstruktive Sozialforschung. Einführung in Methodologie und Praxis qualitativer Forschung. Opladen: Leske und Budrich.

Bolzman, Claudio, Rosita Fibbi und Marie Vial. 2003. Secondas-Secondos. Le processus d'intégration des jeunes adultes issus de la migration espagnole et italienne en Suisse. Zurich: Seismo Verlag.

Bommes, Michael. 2004. Migration, belonging, and the shrinking inclusive capacity of the Nation-State. S. 209-225 in Worlds in the Move : Globalization, Migration, and Cultural Security, hrsg. von Jonathan Friedman und Shalina Randeria. New York: I.B.Tauris.

Bommes, Michael und Jost Halfmann. 1994. Migration und Inklusion. Spannungen zwischen Nationalstaat und Wohlfahrtsstaat. Kölner Zeitschrift für Soziologie und Sozialpsychologie 46: 406-424.

Bös, Mathias und Veronika Schmid. 2012. Staatsbürgerschaft - ein Auslaufmodell? Zur Dialektik der Konstruktion von Mitgliedschaft in nationalstaatlich verfassten Gesellschaften. S. 53-72 in Selbstbeobachtung der modernen Gesellschaft und die neuen Grenzen des Sozialen, hrsg. von Georg Peter und Reuss-Markus Krausse. Wiesbaden: Springer Fachmedien.

Dahinden, Janine. 2010. Wer entwickelt einen transnationalen Habitus? Ungleiche Transnationalisierungprozesse als Ausdruck ungleicher Ressourcenausstattung. S. 83-107 in Die soziale Welt quer 
denken. Transnationalisierung und ihre Folgen für die soziale Arbeit, hrsg. von Christian Reutlinger, Nadja Baghdadi und Johannes Kniffki. Berlin: Frank \& Timme.

D’Amato, Gianni. 2008. Historische und soziologische Übersicht über die Migration in der Schweiz. Schweizerisches Jahrbuch für Entwicklungspolitik 27(2): 177-195.

Fibbi, Rosita, Bülent Kaya und Etienne Piguet. 2003. Le passeport ou le diplôme? Etudes des discriminations à l'embauche des jeunes issus de la migration. Rapport de recherche $31 \mathrm{du}$ Forum suisse pour l'étude des migrations et de la population. Neuenburg: SFM.

Fibbi, Rosita, Mathias Lerch und Philippe Wanner. 2007. Naturalisation and socio-economic characteristics of youth of immigrant descent in Switzerland. Journal of Ethnic and Migration Studies 33(7): 1121-1144.

Frauenfelder, Arnaud. 2007. Les paradoxes de la naturalisation. Enquête auprès de jeunes issus de l'immigration. Paris: L'Harmattan.

Helbling, Marc und Hanspeter Kriesi. 2004. Staatsbürgerverständnis und politische Mobilisierung: Einbürgerungen in Schweizer Gemeinden. Swiss Political Science Review 10(4): 33-58.

Imdorf, Christian. 2010. Wie Ausbildungsbetriebe soziale Ungleichheit reproduzieren: Der Ausschluss von Migrantenjugendlichen bei der Lehrlingsselektion. S. 263-278 in Bildungsungleichheit revisited. Bildung und soziale Ungleichheit vom Kindergarten bis zur Hochschule, hrsg. von Heinz-Hermann Krüger, Ursula Rabe-Kleberg, Rolf-Torsten Kramer und Jürgen Budde. Wiesbaden: VS Verlag für Sozialwissenschaften.

Lister, Ruth, Noel Smith, Sue Middleton und Lynne Cox. 2003. Young people talk about citizenship: empirical perspectives on theoretical and political debates. Citizenship Studies 7(2): 235-253.

Mackert, Jürgen. 2000. Nationalstaat, Staatsbürgerschaft und Immigration: Zur Analyse der Mechanismen interner Schliessung. S. 643-664 in Gute Gesellschaft? Verhandlungen zum 30. Kongress der DGS., hrsg. von Jutta Allmendinger. Opladen: Leske und Budrich.

Mackert, Jürgen. 2006. Staatsbürgerschaft. Eine Einführung. Wiesbaden: VS Verlag für Sozialwissenschaften.

Mackert, Jürgen und Hans-Peter Müller. 2007. Die Staatsbürgerschaft vor postnationalen Herausforderungen. S. 9-27 in Moderne (Staats)Bürgerschaft. Nationale Staatsbürgerschaft und die Debatten der Citizenship Studies, hrsg. von Jürgen Mackert und Hans-Peter Müller. Wiesbaden: Verlag für Sozialwissenschaften.

Marshall, Thomas H. 1992. Bürgerrechte und soziale Klassen. Zur Soziologie des Wohlfahrtsstaats. Herausgegeben, übersetzt und mit einem Vorwort versehen von Elmar Rieger. Frankfurt und New York: Campus Verlag.

Mecheril, P. 2003. Prekäre Verhältnisse. Über natio-ethno-kulturelle (Mehrfach)Zugehörigkeit. Münster: Waxmann.

Mecheril, Paul und Britta Hoffarth. 2006. Zugehörigkeitsordnungen. S. 221-240 in Adoleszenz-Migration - Bildung. Bildungsprozesse Jugendlicher und junger Erwachsener mit Migrationshintergrund, hrsg. von Vera King und Hans-Christoph Koller. Wiesbaden: Verlag für Sozialwissenschaften.

Mey, Eva. 2015. Wege in die Arbeitswelt - dorthin, wo noch Platz ist. Mechanismen und Bedeutung stark fremdbestimmter Berufswahl bei jungen Menschen mit Migrationshintergrund. S. 235-262 in Arbeit, Migration und soziale Arbeit. Prozesse der Marginalisierung in modernen Arbeitsgesellschaften, hrsg. von Thomas Geisen und Markus Ottersbach. Wiesbaden: Springer VS.

Mey, Eva. 2016. Studying second-generation transitions into adulthood in Switzerland: a biographical approach. In Situating Children of Migrants across Borders and Origins: A Methodological Overview, hrsg. von Laura Bernardi, Claudio Bolzman und Jean-Marie Le Goff. Dordrecht: Springer.

Mey, Eva und Miriam Rorato. 2010. Jugendliche mit Migrationshintergrund im Übergang ins Erwachsenenalter - eine biographische Längsschnittstudie (SNF/DORE). Schlussbericht zuhanden des Praxispartners Bundesamt für Migration. 
Mey, Eva, Miriam Rorato und Peter Voll. 2005. Die soziale Stellung der zweiten Generation. Analysen zur schulischen und beruflichen Integration der zweiten Ausländergeneration. S. 61-152 in Eidgenössische Volkszählung 2000, hrsg. von Bundesamt für Statistik. Neuenburg: Bundesamt für Statistik.

Reichel, David. 2011. Staatsbürgerschaft und Integration. Die Bedeutung der Einbürgerung für MigrantInnen. Wiesbaden: VS Verlag, Springer.

Rosenthal, Gabriele. 1995. Erlebte und erzählte Lebensgeschichte. Gestalt und Struktur biographischen Selbstbeschreibungen. Frankfurt a. M.: Campus Verlag.

Schiffauer, Werner. 2004. Cosmopolitans are cosmopolitans: on the relevance of local identification in globalizing society. S. 91-102 in Worlds in the Move: Globalization, Migration, and Cultural Security, hrsg. von Jonathan Friedman und Shalina Randeria. New York: I. B.Tauris.

Schütze, Fritz. 1981. Prozessstrukturen des Lebensablaufes. S. 67-156 in Biographie in handlungswissenschaftlicher Perspektive, hrsg, von Joachim Matthes, Arno Pfeifenberger und Manfred Stosberg. Erlangen: Verlag der Nürnberger Forschungsvereinigung e.V.

Schütze, Fritz. 1983. Biographieforschung und narratives Interview. Neue Praxis 3: 283-294.

Soysal, Yasemin Nuhoglu. 1994. Limits of Citizenship. Migrants and Postnational Membership in Europe. Chicago: University of Chicaco Press.

Walther, Andreas. 2011. Handeln junger Frauen und Männer in der Öffentlichkeit. Partizipation oder Nicht-Partizipation? S. 203-237 in Jugend - Akteurin sozialen Wandels, hrsg. von Axel Pohl, Barbara Stauber und Andreas Walther. Weinheim und München: Juventa.

Wanner, Philippe und Ilka Steiner. 2012. Einbürgerungslandschaft Schweiz. Entwicklungen 1992-2010. Materialien zur Migrationspolitik. Bern: EKM.

Wicker, Hans-Rudolf. 2011. Die neue alte schweizerische Integrationspolitik. S. 11-41 in Wider die Ausgrenzung - für eine offene Schweiz. Band 2: Debatten, hrsg. von Brigitta Gerber und Damir Skenderovic. Zürich: Chronos Verlag.

Wimmer, Andreas. 1998. Binnenintegration und Aussenabschliessung. Die Beziehung zwischen Wohlfahrtsstaat und Migrationssteuerung in der Schweiz des 20. Jahrhunderts. S. 199-221 in Migration im nationalen Wohlfahrtsstaat: theoretische und vergleichende Untersuchungen, hrsg. von Michael Bommes und Jost Halfmann. Osnabrück: Universitätsverlag Rasch.

Witmer, Sandra. 2008. Unterschiedliche Wege zum roten Pass: Ein Vergleich der Einbürgerungsverfahren in den beiden Städten Langenthal und Solothurn. Arbeitsblätter des Instituts für Sozialanthropologie der Universität Bern 43.

Yildiz, Erol. 2010. Die Öffnung der Orte zur Welt und postmigrantische Lebensentwürfe. SWS-Rundschau 50(3): 318-339.

Yildiz, Erol und Marc Hill. 2015. Nach der Migration. Postmigrantische Perspektiven jenseits der Parallelgesellschaft. Bielefeld: transcript. 


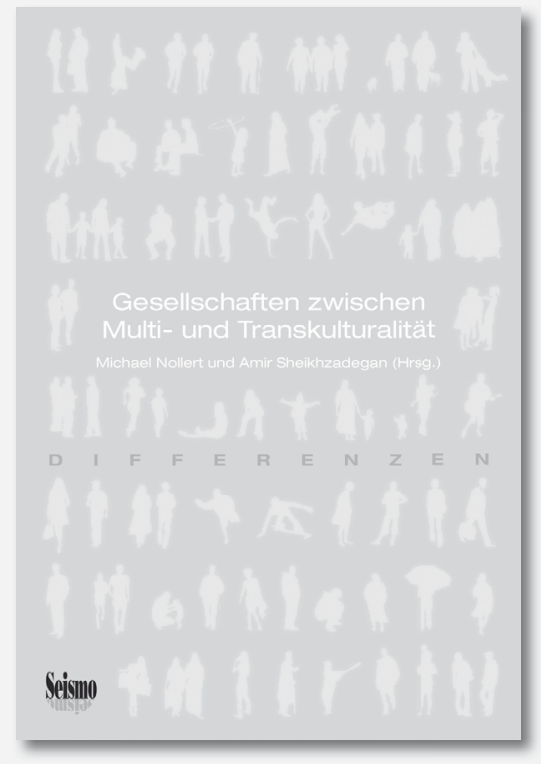

Die zunehmende Immigration aus islamisch geprägten Staaten stellt eine kulturelle und sozialpolitische Herausforderung dar. Kulturelle Differenz wird dabei unter unterschiedlichen theoretischen Prämissen diskutiert: Vertreterlnnen des Multikulturalismus gehen davon aus, dass ein Zusammenleben verschiedener Kulturen nicht nur wünschbar, sondern auch möglich ist. "Transkulturalität» impliziert hingegen, dass Kulturen keine homogene, abgrenzbare Entitäten sind, und es im Hinblick auf soziale Kohäsion auch nicht wünschbar ist, dass soziale Gruppen kulturelle Differenzen akzentuieren oder gar politisch instrumentalisieren.

\section{Michael Nollert und Amir Sheikhzadegan (Hrsg.) Gesellschaften zwischen Multi- und Transkulturalität}

208 Seiten

ISBN 978-3-03777-151-8

SFr. 38.-/Euro 34.-

Michael Nollert ist Professor für Sozialforschung und Sozialpolitik im Studienbereich Soziologie, Sozialpolitik und Sozialarbeit der Universität Fribourg.

Amir Sheikhzadegan ist Doktorassistent im Studienbereich Soziologie, Sozialpolitik und Sozialarbeit der Universität Fribourg. 